\title{
Circular RNAs in Cardiovascular Disease: An Overview
}

\author{
Ximin Fan, ${ }^{1}$ Xinyu Weng, ${ }^{1}$ Yifan Zhao, ${ }^{1}$ Wei Chen, ${ }^{1}$ Tianyi Gan, ${ }^{2}$ and Dachun Xu ${ }^{1}$ \\ ${ }^{1}$ Department of Cardiology, Shanghai Tenth People's Hospital, Tongji University School of Medicine, Shanghai, China \\ ${ }^{2}$ State Key Laboratory of Cardiovascular Disease, Heart Failure Center Fuwai Hospital, National Center for Cardiovascular Diseases, \\ Chinese Academy of Medical Sciences and Peking Union Medical College, Beijing, China \\ Correspondence should be addressed to Dachun Xu; xdc77@aliyun.com
}

Received 29 August 2016; Accepted 13 December 2016; Published 22 January 2017

Academic Editor: Xinli Li

Copyright (C) 2017 Ximin Fan et al. This is an open access article distributed under the Creative Commons Attribution License, which permits unrestricted use, distribution, and reproduction in any medium, provided the original work is properly cited.

\begin{abstract}
Circular RNA (circRNA), a novel type of endogenous noncoding RNA (ncRNA), has become a research hotspot in recent years. CircRNAs are abundant and stably exist in creatures, and they are found with covalently closed loop structures in which they are quite different from linear RNAs. Nowadays, an increasing number of scientists have demonstrated that circRNAs may have played an essential role in the regulation of gene expression, especially acting as miRNA sponges, and have described the potential mechanisms of several circRNAs in diseases, hinting at their clinical therapeutic values. In this review, the authors summarized the current understandings of the biogenesis and properties of circRNAs and their functions and role as biomarkers in cardiovascular diseases.
\end{abstract}

\section{Introduction}

Circular RNA (circRNA) has been recently discovered and is becoming a research focus in the field of untranslated RNA. Circular RNAs are a kind of competing endogenous RNAs (ceRNA) [1] which can regulate RNAs. Currently, there are two types of ceRNAs including coding and noncoding RNAs. The latter includes long noncoding RNAs (lncRNA), microRNAs (miRNA), and circular RNAs (circRNA) [2]. CircRNAs are predominantly found in cytoplasm and are highly stable in human bodies. They are also identified in various organisms [3-6] and are abundantly expressed and evolutionarily conserved across the eukaryotic tree of life $[7,8]$, especially in human and mice [7]. In this review, we have summarized the current studies of the biogenesis, the properties and the functions of circRNAs, and their roles in cardiovascular diseases.

\section{Biogenesis of CircRNAs}

CircRNAs mainly come from the exons of protein-coding genes, and they are not supposed to be produced by a normal mode of RNA splicing [9]. They differ structurally from other RNAs in that they are circularized by joining the $3^{\prime}$ and $5^{\prime}$ ends together via exon circularization or intron circularization [10]. Jeck and his colleagues have proposed two different models of exon circularization of circRNA: "lariat-driven circularization" (Figure 1(a)) and "intron-pairing-driven circularization" (Figure 1(b)) [11]. The former is associated with exon skipping, in which one or more exons of the transcript are skipped, and it contributes to an exon-containing lariat. The lariat itself would then be joined by spliceosome and become an exon circle. The latter is mostly related to complementary motifs, which are present in the intronic regions. Accumulated evidence has verified that pairing between these motifs induces circularization [11]. The genome-wide analyses observed that complementary flanking Alu elements are important for the formation of circRNAs [12]. However, Alu elements are not specifically needed if there are other inverted repeats [13]. That is to say circRNA can also be generated via direct base-pairing in the presence of inverted repeat without Alu elements. Nevertheless, more than half of the known circRNAs do not have complementary flanking in the intronic sequences, as it has been reported that $38 \%$ of all known Caenorhabditis elegans circRNAs and $9 \%$ of all known human circRNAs can be recognized using the base-pairing potential to cyclize the exons [14]. In these cases, their introns were found to be 


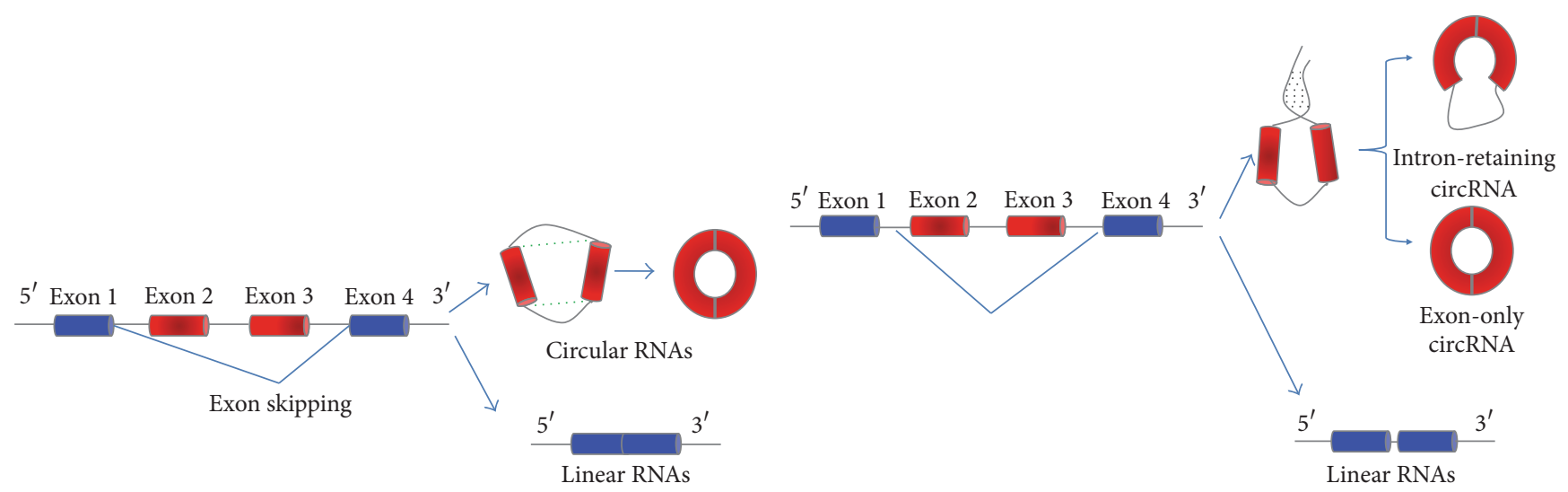

(a)

(b)

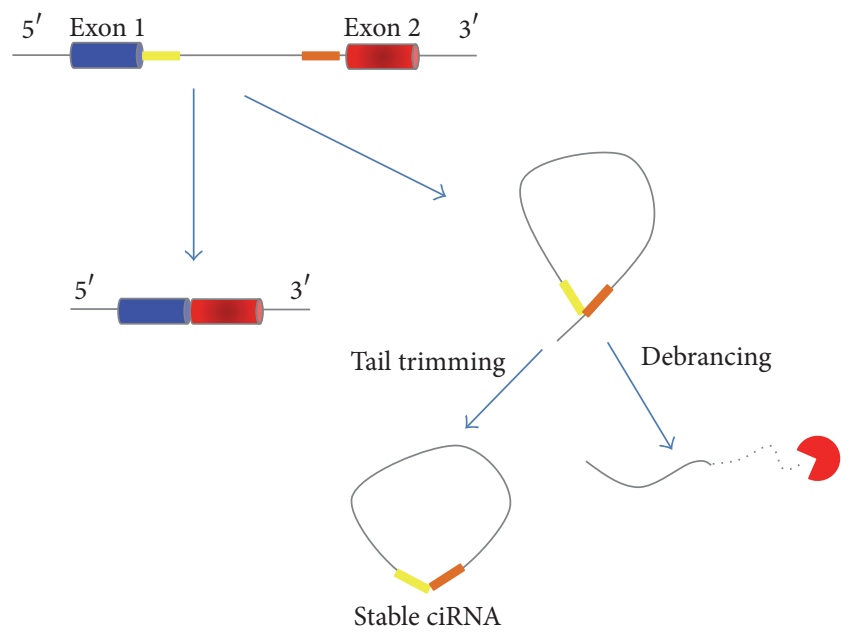

(c)

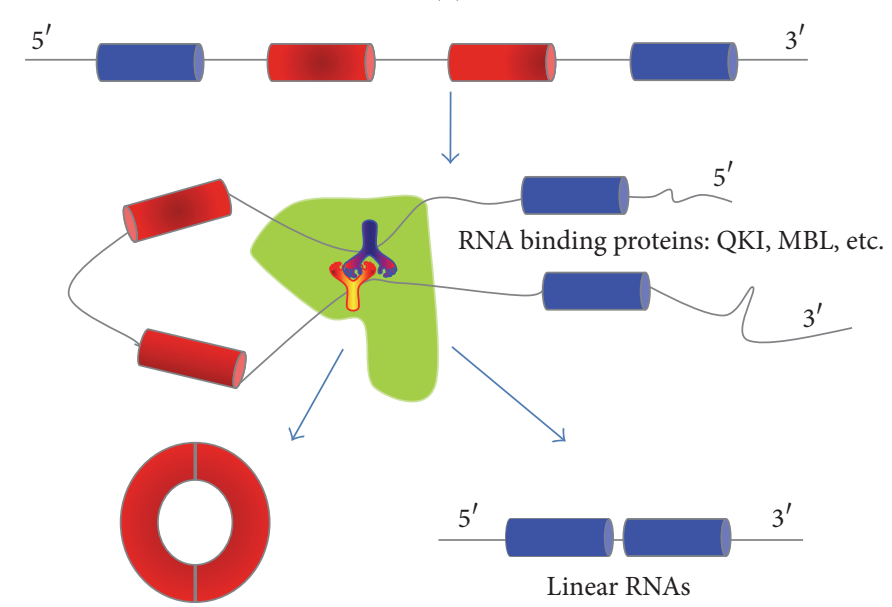

Circular RNAs

(d)

FIGURE 1: Models of circRNA biogenesis: (a) lariat-driven circularization: exon skipping leads to a covalent splice of the splice donor in $3^{\prime}$ end of exon 1 to the splice acceptor in $5^{\prime}$ end of exon 4 , which forms a linear product and a lariat structure containing the skipped exons 2 and 3. Then the lariat is joined by spliceosome and the introns are removed to form a circRNA. (b) Intron-pairing-driven circularization: intron 1 and intron 3, which contain complementary sequence motifs, lead to close proximity through direct base-pairing and form a linear RNA and a circular structure. The splicing of the two introns produces a circRNA (exon-only circRNA), while the generation of an EIciRNA (intron-retaining circRNA) is caused by the presence of a retained intron. (c) Circular intronic RNAs: the existence of $7 \mathrm{nt}$ GU-rich element near exon 1 (yellow box) and $11 \mathrm{nt}$ C-rich element near exon 2 (orange box) makes it possible for an intron to escape debranching when the intron lariat is produced from the splicing reaction. (d) RNA binding proteins (RBPs) driven circularization: the interaction between RBPs (Y-shape) can bind to sequence motifs and bring two flanking introns close together. Then the introns are removed to form a circRNA.

remarkably longer than average $[15,16]$. After two introns forming circular structure via base-pairing, the two introns are removed or retained to form a circRNA (exon-only circRNA) or an EIciRNA (intron-retaining circRNA) [11, 17]. The intron-retaining circRNAs were found recently by Li et al. who termed them exon-intron circRNAs or EIciRNAs [17]. However, the mechanism of their formation is not clear yet. Soon after Jeck, Zhang et al. proposed a model of circular intronic RNAs (ciRNAs), suggesting that circRNAs could also come from lariat introns that escape debranching. The generation of ciRNA is related to a $7 \mathrm{nt}$ GU-rich element near the $5^{\prime}$ splicing site and an $11 \mathrm{nt} \mathrm{C}$-rich element near the branching point (Figure 1(c)) [18]. Judging from the above findings, circRNAs can also arise from introns. Recent studies have also suggested another pathway of circRNA biogenesis through RNA binding proteins (RBPs). Quaking protein $(\mathrm{QKI})$ and Muscleblind protein (MBL) can bind certain circRNA flanking introns and act as RBPs to bring two flanking intronic sequences close together, hence provoking the circularization (Figure 1(d)) [19, 20].

\section{Properties of CircRNAs}

There are several important properties of circRNAs. First, they are widely expressed in human cells, and their expression levels are more than 10 times higher than those of the corresponding linear mRNAs $[9,11]$. Second, different from traditional linear RNAs, circRNAs form covalently closed loop structures with neither $5^{\prime}-3^{\prime}$ polarities nor polyadenylated tails, resulting in less degradation by RNA exonuclease 
or RNase R, which makes them more stable than linear RNAs in human bodies $[21,22]$. Third, besides a few circRNAs, most of them have highly conserved sequences between species [9, 11, 12, 22]. Fourth, the vast majority of them reside in cytoplasm [23], while only a small portion is in cell nucleus [12]. Fifth, they primarily come from exons, while a few others come from introns or intron fragments. Sixth, some circRNAs have microRNA response element (MRE), through which they can have interactions with miRNAs, thus regulating target gene expressions [13, 24]. Seventh, most circRNAs are endogenous noncoding RNAs (ncRNA) [9]. Eighth, the majority of circRNAs can play regulatory roles in transcription and posttranscription, and only a few play roles in transcription [18]. Ninth, similar to linear mRNAs, circRNAs also show tissue-specific and/or developmentalstage-specific expression $[8,25]$.

\section{Function of CircRNAs}

As circRNAs are abundant and evolutionarily conserved, several potential functions of circRNAs have been predicted $[10,26]$. Some studies have revealed that circRNAs could function as microRNA (miRNA) sponges, modulate alternative splicing or transcription, and regulate the expression of parental genes $[8,13,17,18,20]$, among which the function as miRNA sponges has been a focus of recent research.

4.1. CircRNAs Function as MiRNA Sponges. CircRNAs are a kind of competing endogenous RNAs (ceRNA), which contain shared miRNA response elements (MRE) [27]. Therefore, the presence or absence of circRNAs would affect the activities of miRNAs. CircRNAs can competitively bind to miRNAs, which results in the reduction of miRNA molecules. Then the reduced miRNAs would have less inhibiting effects on miRNA target genes, resulting in the upregulation of the expression of miRNA target genes [8]. As circRNAs have many miRNA binding sites and can absorb miRNAs like a sponge, they are initially demonstrated as miRNA sponges $[8,13]$.

The most representative circRNA is Cdrlas (antisense to the cerebellar degeneration-related protein 1 transcript) [28], also termed as ciRS-7 (circular RNA sponge for miR-7), and Cdrlas has a negative regulation of the activity of miR-7 [13]. According to the bioinformatics, Cdrlas contains more than 70 binding sites for miR-7 and is bound by Argonaute proteins and functions as a miR-7 sponge in brain tissues [13], islet cells [29], and some other cells. The expressions of published miR-7 target genes $[8,13]$, such as SNCA, EGFR, and IRS2 [30-32], are downregulated by silencing of Cdr2as [13]. Some other studies also demonstrated that miR-7 can regulate the occurrence of various cancers through repressing its target genes like EGFR (epidermal growth factor receptor) [33], ACK1 (Activated Cdc42-associated Tyrosine Kinase 1) [34], or IGF1R (insulin-like growth factor 1 receptor) [35]. Similar to Cdrlas, murine sex-determining region Y (Sry) is another recognized circRNA with 16 biding sites for miR138 and is likely to act as a miR-138 sponge [13]. Hence, the findings that circRNAs can interact with miRNAs and alter the expression of miRNAs and/or its target genes $[8,13]$ may have great significance in the pathogenesis research for related diseases and may point out a potential direction of future treatment as well.

4.2. CircRNAs Modulate Alternative Splicing or Transcription. $\mathrm{MBL}$ protein is a splicing factor which can affect alternative mbl pre-mRNA splicing during generation of mbl mRNA and circular $\mathrm{Mbl}$ ( $\operatorname{circMbl}$ ). CircMbl derived from the $\mathrm{mbl}$ locus has conserved binding sites for the MBL protein in the flanking intronic sequences. Reut Ashwal-Fluss has verified that MBL levels can strongly affect circMbl biosynthesis when MBL proteins bind to both introns of the circMbl simultaneously, rather than the large total number of $\mathrm{MBL}$ binding sites on the circMbl alone. Moreover, a balance between circMbl biogenesis and splicing has been proposed. When MBL is in excess, mbl pre-mRNA will produce more circMbl and less mbl mRNA, resulting in the decrease of $\mathrm{MBL}$ levels, because more circMbl binds to more MBL while less mRNA produces less MBL (Figure 2) [20]. These findings may hint that some circRNA may play a role in controlling the expression of mRNA through binding to RNA binding proteins (RBPs) and affecting the canonical splicing.

As stated above, the three types of circRNAs, namely, ciRNA, circRNA, and EIciRNA, resulted from the competition of complementary flanking in or across the intronic sequences. For those circRNAs and the corresponding linear mRNAs which are generated from a single gene locus, their progressions are closely related, as complementary intronic sequences can lead to more linear mRNA generation, while complementary sequences flanking across the intronic sequences can promote exon circularization. Therefore, circRNAs are interrelated with each other, and the competitive balance among them can affect the expression of mRNA $[12,36]$.

4.3. CircRNAs Regulate Parental Gene Expression. EIciRNAs and ciRNAs were recently revealed to promote transcription of the parental genes. Zhang and others found that ciRNAs have few microRNA binding sites, suggesting that they may function differently [37]. Some ciRNAs are predominantly localized in the nucleus and can promote host-gene transcription through interacting with the RNA polymerase II (pol II) in the promoter region of genes (Figure 3(a)) [18, 38]. EIciRNAs are also abundant in nucleus, such as circEIF3J and circPAIP2, and can bind to U1 snRNP (small nuclear ribonucleoproteins) in nucleus to form EIciRNA-U1 snRNP complexes. The complexes can interact with RNA polymerase II in the promoter region of genes, therefore enhancing the transcription of their parental genes (Figure 3(b)) $[17,38]$. In some cases, when both circRNA and $3^{\prime}$-untranslated region (UTR) of the transcript from their parental gene share the same miRNA binding sites, circRNA can act as miRNA sponge in the cytoplasm to improve the translations of the transcript from their parental gene. Li and his colleagues found that cir-ITCH share the same miRNA (miR-7, miR17 , and miR-214) binding sites with $3^{\prime}$-UTR of ITCH mRNA and increase ITCH protein levels [39]. Here, we speculate that intronic circRNAs may be efficient for regulatory functions in the cell nucleus close to the transcription site, while the 


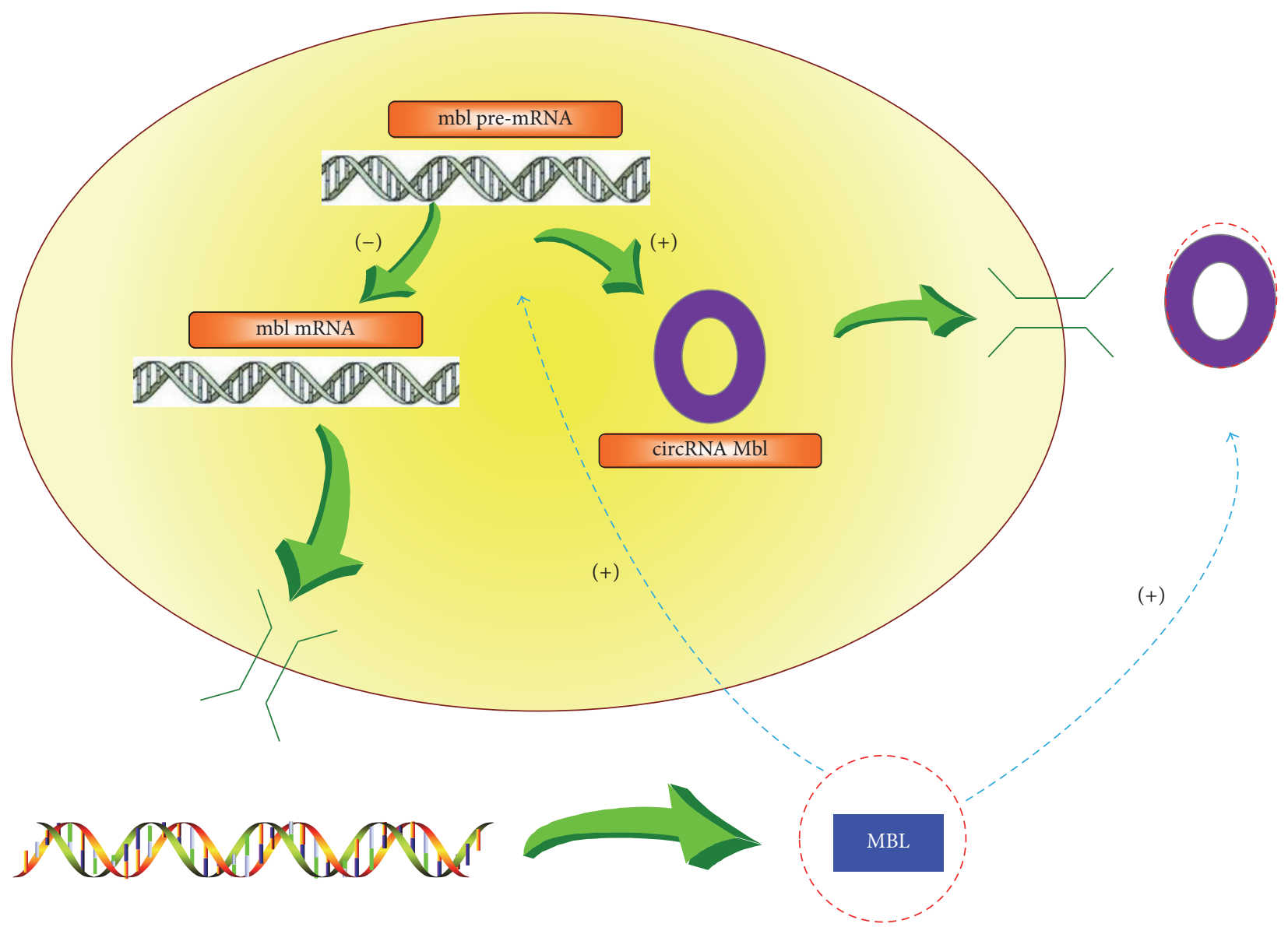

Figure 2: A model of the generation of circular Mbl (circMbl) and MBL: CircMbl is derived from the mbl locus and has conserved binding sites for the MBL protein in the flanking intronic sequences, so it can bind MBL protein. Besides, BML levels can strongly affect the splicing process when mbl pre-mRNA produces mbl mRNA and circMbl. When the level of MBL is high, mbl pre-mRNA produces less mbl mRNA and more circMbl. Less mbl mRNA generates less MBL and more circMbl binds to more MBL, so that MBL level decreases. (+) represents promotion while $(-)$ represents inhibition in this figure, and vice versa.

generalized exon-only circRNAs may fulfill transcriptional regulation in cytoplasm [36].

\section{Roles in Cardiovascular Diseases}

Several recent studies have suggested that circRNAs may play essential roles in the initiation and development of cardiovascular diseases (Table 1).

\subsection{Circular RNA HRCR Protects Heart from Pathological} Hypertrophy and Heart Failure. MiR-223 is an endogenous regulator which can induce cardiac hypertrophy and heart failure [44], as well as hypertrophy in cardiomyocytes [45]. ARC (apoptosis repressor with CARD domain) is a downstream target of miR-223 [46], indicating that miR-223 exerts its effect through ARC. It has been reported that ARC play a protective role in cardiomyocyte hypertrophy and apoptosis [47-49]. As circRNAs act as miRNA sponges to interact with miRNAs and influence the expression of miRNA [8, 13], Wang and his team proposed and verified that the heartrelated circRNA (HRCR) can directly bind to miR-223 and act as an endogenous miR-223 sponge to inhibit miR-223 activity, which results in the increase of ARC expression [46]. Taken together, HRCR acts as an endogenous miR-223 sponge to modulate the expression of miR-223 and ARC, through which it regulates cardiomyocytes hypertrophy as well as cardiac hypertrophy and heart failure. Thus, it is speculated that enforced expression of HRCR attenuates the development of cardiac hypertrophy and heart failure. Furthermore, it may be targeted for drug development in the inhibition of related cardiovascular disorders.

5.2. Circular RNA Cdrlas Induces Myocardial Infarction. Myocardial infarction (MI) has been one of the leading causes of death and disability around the world [50]. During MI development, prolonged myocardial ischemia contributes to the myocardial cell death process [51], namely, apoptosis, one programmed cell death.

Recently, one of the circRNAs, Cdrlas, was described to function as miR-7 sponges and can inhibit the activity of miR-7 $[8,13]$. Zhao and his colleagues proposed that miR$7 \mathrm{a} / \mathrm{b}$ can act as protective roles in myocardial cells through 


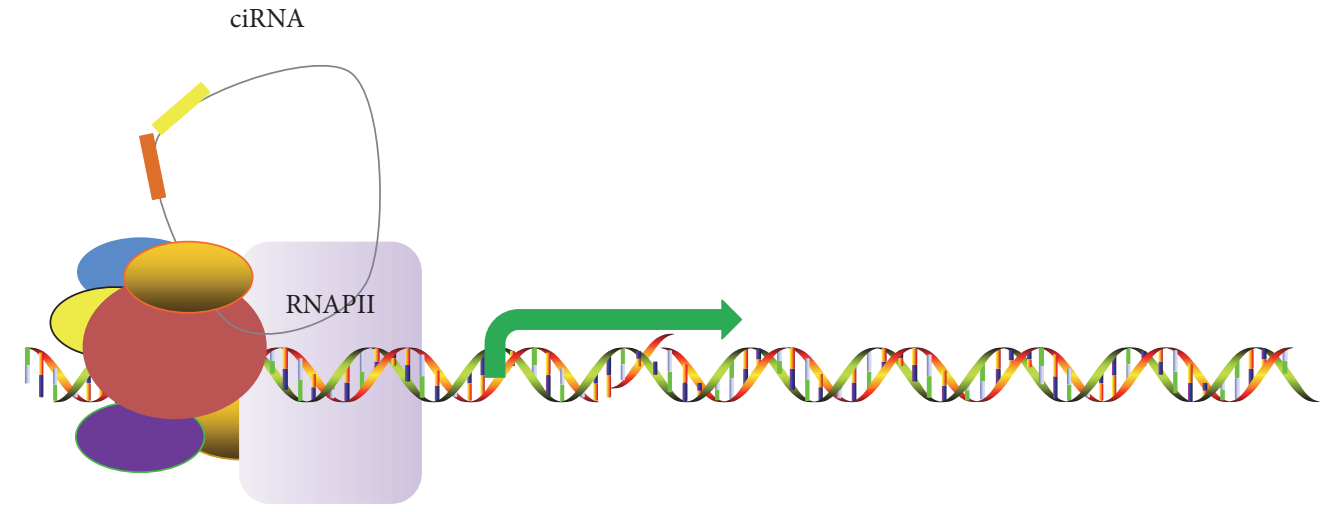

(a)
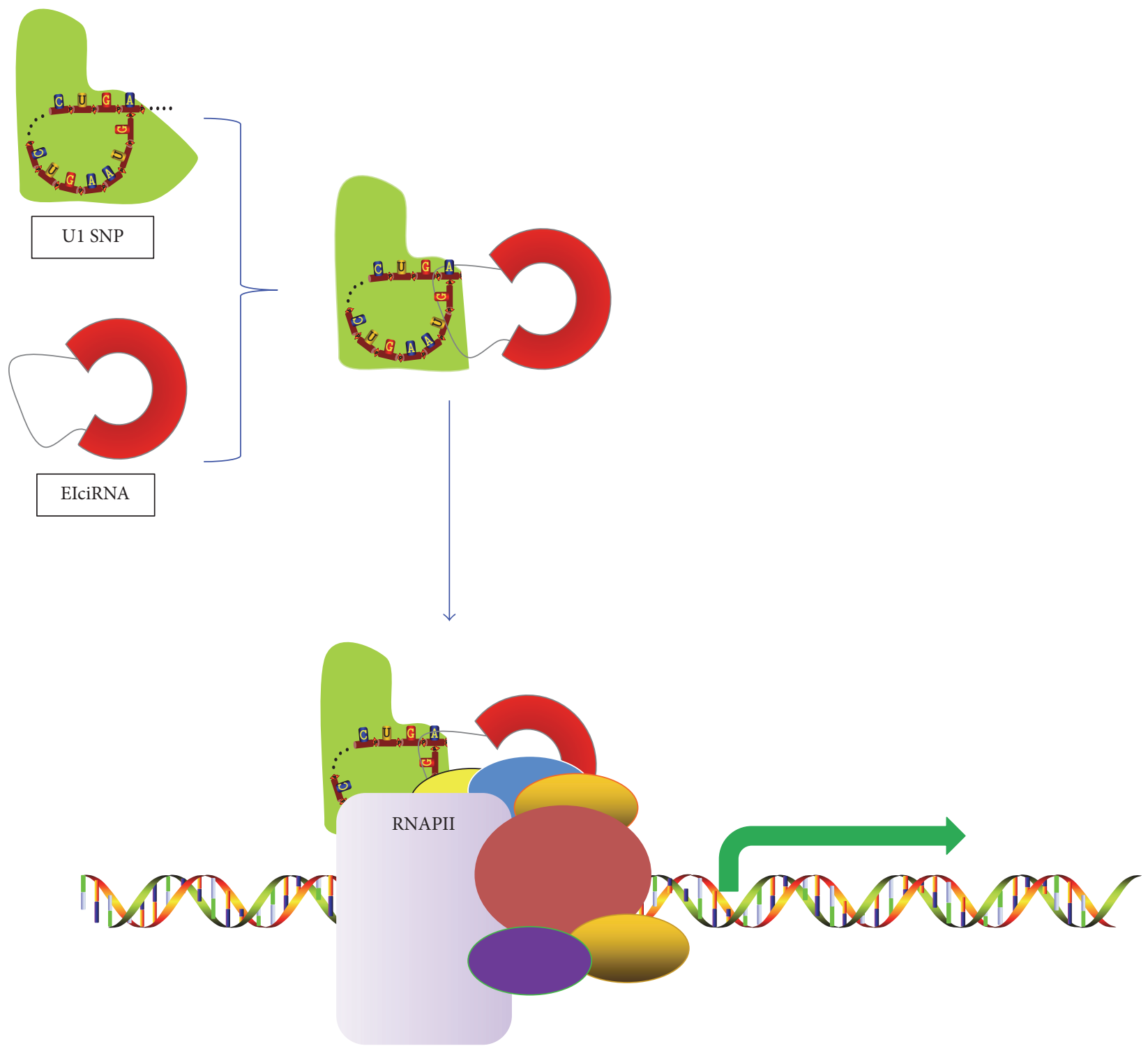

(b)

FIGURE 3: Model of circRNA regulating the expression of a parental gene: (a) CiRNAs come from lariat introns that escape debranching. CiRNA can bind to RNA polymerase II (RNA pol II) and function in the promoter region of genes. (b) EIciRNA can interact with the U1 snRNP (small nuclear ribonucleoprotein) via specific RNA-RNA interaction between U1 snRNP and EIciRNA to form EIciRNA-U1 snRNP complexes, and the complexes will recruit RNA pol II to the promoter to stimulate host gene expression. 
TABLE 1: An overview of circular RNAs in various cardiovascular diseases.

\begin{tabular}{|c|c|c|c|c|c|}
\hline & Diseases type & CircRNA & Targets & Effect on diseases & References \\
\hline $\mathrm{a}$ & Heart failure and pathological hypertrophy & HRCR & miR-223 ARC & Suppress & {$[40]$} \\
\hline $\mathrm{b}$ & Myocardial Infarction & Cdrlas(ciRS-7) & miR-7 SP1, PARP & Induce & {$[41]$} \\
\hline c & Cardiac senescence & circ-Foxo3 & ID1, E2F1, FAK, HIF1a & Induce & {$[42]$} \\
\hline $\mathrm{d}$ & Atherosclerosis & cANRIL & The INK4/ARF locus & Regulate & [43] \\
\hline
\end{tabular}

a: HRCR can act as miRNA sponges and bind to miR-223. ARC, a kind of protein, is the downstream target of miR-223, so that HRCR can enhance ARC's protective role as blocking the progression of cardiac pathological hypertrophy and heart failure through inhibiting miR-223 activity. b: Cdrlas can function as miR-7 sponges. SP1 and PARP are miR-7 target genes and can inhibit miR-7-induced protective role during MI development. c: Circ-Foxo3 can hinder transcription factors' (ID1, E2F1, FAK, and HIFla) transfer into nucleus so as to repress their antiageing effect. d: CANRIL is an antisense transcript from the INK4/ARF locus resulting from alternative ANRIL transcription and splicing, while cANRIL can also influence the PcG-mediated INK4/ARF silencing. As SNPs on chromosome 9p21 near the INK4/ARF locus can control INK4/ARF expression, which is correlated to atherosclerosis susceptibility, cANRIL can have a regulatory role here.

negatively regulating PARP and decreasing apoptosis [52]. SP1 and PARP (poly ADP-ribose polymerase) are miR-7 target genes [53] and can inhibit miR-7a-induced decrease of cell apoptosis under hypoxia treatment [53] and play proapoptotic roles during MI development [54, 55]. Therefore, Cdrlas can function as a miR-7a sponge in promoting MI injuries through reducing the activity of miR-7a and upgrading the expression of miR-7a targets like PARP and SP1, which indicates the key role of Cdrlas/miR-7a axle in MIinduced myocardial apoptosis.

In terms of this new finding that Cdrlas functions as a miR-7a sponge in myocardial cells and regulates miR-7a, PARP, and SP1 in MI injury, further efforts can be done to move forward and figure out the potential therapeutic value for improving MI-related injuries.

5.3. Circular RNA Circ-Foxo3 Promotes Cardiac Senescence. Circ-Foxo3 is derived from a member of the forkhead family of transcription factors which is called Foxo3 and has been detected to be highly expressed in the cytoplasm of aged mice and patients [56]. A number of researches have proved that the high expressions of circ-Foxo3 accompanied with more cells were held up at G1 phase and unable to transit to $S$ phase. Based on the previous findings, Du et al. proposed that the expression of the circ-Foxo3 represses cell proliferation and cell cycle progression [57].

Transcription factors ID1, E2F1, FAK, and HIF1a play an antisenescence role while entering cell nucleus, but circFoxo 3 could repress this antiageing effect by hindering their transfer to nucleus. The gain-and-loss of function experiment also demonstrated a positive relation between circ-Foxp 3 and senescence [56].

Therefore, the ectopic expression of circ-Foxo3 is supposed to promote senescence through arresting and relocating ID1, E2F1, FAK, and HIFla in cytoplasm and blocking their antisenescent function. These findings may provide new insights into the inhibition of cardiac senescence and myocardial protection.

5.4. Circular RNA cANRIL Is Correlated with Atherosclerosis Risk. Several genome-wide association studies (GWAS) have revealed the links between single nucleotide polymorphisms (SNPs) on chromosome 9p21 near the INK4/ARF locus and ASVD [58, 59], suggesting that chromosome 9p21.3 is correlated to the susceptibility of ASVD. Liu and his colleagues verified that the ASVD-associated SNPs at 9p21 can control INK4/ARF (cyclin-dependent kinase 4 inhibitor, INK4a; alternative reading frame, ARF) expression [60]. Circular ANRIL RNA (circular antisense noncoding RNA in the INK4 locus, cANRIL) is an antisense transcript from the INK4A/ARF locus [61]. SNPs on chromosome 9p21.3 near the INK4/ARF locus regulate INK4/ARF transcription through modulating ANRIL splicing and cANRIL production [40], indicating that the structure and expression of CANRIL species resulted from the alternative splicing in the process of INK4/ARF transcription. Jacobs and colleagues figured out that the INK4/ARF locus can be inhibited by Polycomb group (PcG) complexes [62]. As cANRIL can influence the PcG-mediated INK4/ARF silencing through recruiting PcG complexes [63-65], Burd and his colleagues speculated that modified cANRIL structure can result in changes in PcGmediated INK4/ARF silencing and atherosclerosis susceptibility [40]. They also suggested that cANRIL expression may be a useful marker which is of pathogenic relevance to ASVD susceptibility [40].

\section{Conclusion}

CircRNAs are now a noticeable area in the field of RNA. It is clear that natural circRNAs are an abundant, diverse, stable, and conserved class of RNA molecules, representing a new type of regulatory noncoding RNA. Nevertheless, their biological roles are not yet clearly understood, as well as their localization and degradation. Recent researches have demonstrated that circRNAs can act as sponges to bind to miRNAs, regulate transcription, or affect gene expression, and we believe that there might be other functions remaining to be revealed. Recently, a circRNA database has been constructed (http://circnet.mbc.nctu.edu.tw/) [66]. This database provides tissue-specific circRNA expression profiles and circRNA-miRNA-gene regulatory networks [66].

As is known to all, miRNAs regulate cardiac function through regulating the development, differentiation, proliferation, and apoptosis of cells during the progression of diseases [41, 67, 68]. Besides, abnormal expression of miRNA may induce cardiac fibrosis through targeting TGF$\beta$, including miR-378, miR-122, miR-29, and miR-26 [69]. Moreover, one miRNA can have many target mRNA [42, 70], while one mRNA can be managed by several miRNAs [71]. In this review, we summarized recent studies on the 
interaction of circRNAs with others and the relationships between circRNAs and cardiovascular diseases, hinting that circRNAs play special regulating roles in the initiation and progression of related diseases. We noted two circRNAs, HRCR and Cdrlas, which could function as miRNA sponges in the cardiovascular diseases, indicating that some circRNAs may act as upstream regulators of miRNAs. As roles of many miRNAs in different cardiovascular diseases have been verified [72-75] and several circRNAs have been detected in the myocardium of humans and mice [43], we think more studies can be done in the future to figure out the link of circRNAs and miRNAs. Therefore, we speculate that circRNAs may serve as diagnostic or prognostic biomarkers of disease and have potential therapeutic values. However, more studies have to be done before we fully understand the biological and molecular mechanisms of circRNAs in the development of cardiovascular diseases. Perhaps in the future, circRNAs may have great potential in clinical diagnosis and treatment of disease. With the development of new technologies, more circRNAs will be detected as well as their new functions.

\section{Competing Interests}

The authors declare that there is no conflict of interests regarding the publication of this paper.

\section{Authors' Contributions}

Ximin Fan and Xinyu Weng have contributed equally to this work.

\section{Acknowledgments}

The work was not supported by any grant/fund. Costs to publish in open access were covered by the department of Cardiology, Shanghai Tenth People's Hospital, Shanghai, China.

\section{References}

[1] J. Li, J. Yang, P. Zhou, Y. Le, and Z. Gong, "The biological functions and regulations of competing endogenous RNA," $Y i$ Chuan, vol. 37, no. 8, pp. 756-764, 2015.

[2] J. Beermann, M.-T. Piccoli, J. Viereck, and T. Thum, "Noncoding rnas in development and disease: background, mechanisms, and therapeutic approaches," Physiological Reviews, vol. 96, no. 4, pp. 1297-1325, 2016.

[3] H. L. Sanger, G. Klotz, D. Riesner, H. J. Gross, and A. K. Kleinschmidt, "Viroids are single stranded covalently closed circular RNA molecules existing as highly base paired rod like structures," Proceedings of the National Academy of Sciences of the United States of America, vol. 73, no. 11, pp. 3852-3856, 1976.

[4] A. Kos, R. Dijkema, A. C. Arnberg, P. H. Van Der Meide, and H. Schellekens, "The hepatitis delta $(\delta)$ virus possesses a circular RNA," Nature, vol. 323, no. 6088, pp. 558-560, 1986.

[5] B. Capel, A. Swain, S. Nicolis et al., "Circular transcripts of the testis-determining gene Sry in adult mouse testis," Cell, vol. 73, no. 5, pp. 1019-1030, 1993.
[6] M. Danan, S. Schwartz, S. Edelheit, and R. Sorek, "Transcriptome-wide discovery of circular RNAs in Archaea," Nucleic Acids Research, vol. 40, no. 7, pp. 3131-3142, 2012.

[7] P. L. Wang, Y. Bao, M. C. Yee et al., "Circular RNA is expressed across the eukaryotic tree of life," PLOS ONE, vol. 9, no. 6, Article ID e90859, 2014

[8] S. Memczak, M. Jens, A. Elefsinioti et al., "Circular RNAs are a large class of animal RNAs with regulatory potency," Nature, vol. 495, no. 7441, pp. 333-338, 2013.

[9] J. Salzman, C. Gawad, P. L. Wang, N. Lacayo, and P. O. Brown, "Circular RNAs are the predominant transcript isoform from hundreds of human genes in diverse cell types," PLoS ONE, vol. 7, no. 2, Article ID e30733, 2012.

[10] W. R. Jeck and N. E. Sharpless, "Detecting and characterizing circular RNAs," Nature Biotechnology, vol. 32, no. 5, pp. 453-461, 2014.

[11] W. R. Jeck, J. A. Sorrentino, K. Wang et al., "Circular RNAs are abundant, conserved, and associated with ALU repeats," RNA, vol. 19, no. 2, pp. 141-157, 2013.

[12] X.-O. Zhang, H.-B. Wang, Y. Zhang, X. Lu, L.-L. Chen, and L. Yang, "Complementary sequence-mediated exon circularization," Cell, vol. 159, no. 1, pp. 134-147, 2014.

[13] T. B. Hansen, T. I. Jensen, B. H. Clausen et al., "Natural RNA circles function as efficient microRNA sponges," Nature, vol. 495, no. 7441, pp. 384-388, 2013.

[14] A. Ivanov, S. Memczak, E. Wyler et al., "Analysis of intron sequences reveals hallmarks of circular RNA biogenesis in animals," Cell Reports, vol. 10, no. 2, pp. 170-177, 2015.

[15] J. O. Westholm, P. Miura, S. Olson et al., "Genome-wide analysis of Drosophila circular RNAs reveals their structural and sequence properties and age-dependent neural accumulation," Cell Reports, vol. 9, no. 5, pp. 1966-1980, 2014.

[16] K. K. Ebbesen, J. Kjems, and T. B. Hansen, "Circular RNAs: identification, biogenesis and function," Biochimica et Biophysica Acta (BBA)_Gene Regulatory Mechanisms, vol. 1859, no. 1, pp. 163-168, 2016.

[17] Z. Li, C. Huang, C. Bao et al., "Exon-intron circular RNAs regulate transcription in the nucleus," Nature Structural and Molecular Biology, vol. 22, no. 3, pp. 256-264, 2015.

[18] Y. Zhang, X.-O. Zhang, T. Chen et al., "Circular intronic long noncoding RNAs," Molecular Cell, vol. 51, no. 6, pp. 792-806, 2013.

[19] S. J. Conn, K. A. Pillman, J. Toubia et al., "The RNA binding protein quaking regulates formation of circRNAs," Cell, vol. 160, no. 6, pp. 1125-1134, 2015

[20] R. Ashwal-Fluss, M. Meyer, N. R. Pamudurti et al., "CircRNA biogenesis competes with pre-mRNA splicing," Molecular Cell, vol. 56, no. 1, pp. 55-66, 2014.

[21] H. Suzuki and T. Tsukahara, "A view of pre-mRNA splicing from RNase R resistant RNAs," International Journal of Molecular Sciences, vol. 15, no. 6, pp. 9331-9342, 2014.

[22] H. Suzuki, Y. Zuo, J. Wang, M. Q. Zhang, A. Malhotra, and A. Mayeda, "Characterization of RNase R-digested cellular RNA source that consists of lariat and circular RNAs from pre-mRNA splicing," Nucleic Acids Research, vol. 34, no. 8, article e63, 2006.

[23] C. Cocquerelle, B. Mascrez, D. Hetuin, and B. Bailleul, "Missplicing yields circular RNA molecules," FASEB Journal, vol. 7, no. 1, pp. 155-160, 1993.

[24] C. Yang, D. Wu, L. Gao et al., "Competing endogenous RNA networks in human cancer: hypothesis, validation, and perspectives," Oncotarget, vol. 7, no. 12, pp. 13479-13490, 2016. 
[25] J. U. Guo, V. Agarwal, H. Guo, and D. P. Bartel, "Expanded identification and characterization of mammalian circular RNAs," Genome Biology, vol. 15, no. 7, article no. 409, 2014.

[26] M. W. Hentze and T. Preiss, "Circular RNAs: splicing's enigma variations," EMBO Journal, vol. 32, no. 7, pp. 923-925, 2013.

[27] L. Salmena, L. Poliseno, Y. Tay, L. Kats, and P. P. Pandolfi, "A ceRNA hypothesis: the Rosetta Stone of a hidden RNA language?" Cell, vol. 146, no. 3, pp. 353-358, 2011.

[28] T. B. Hansen, E. D. Wiklund, J. B. Bramsen et al., "miRNAdependent gene silencing involving Ago2-mediated cleavage of a circular antisense RNA," The EMBO Journal, vol. 30, no. 21, pp. 4414-4422, 2011.

[29] H. Xu, S. Guo, W. Li, and P. Yu, "The circular RNA Cdrlas, via miR-7 and its targets, regulates insulin transcription and secretion in islet cells," Scientific Reports, vol. 5, Article ID 12453 , 2015.

[30] E. Junn, K.-W. Lee, S. J. Byeong, T. W. Chan, J.-Y. Im, and M. M. Mouradian, "Repression of $\alpha$-synuclein expression and toxicity by microRNA-7," Proceedings of the National Academy of Sciences of the United States of America, vol. 106, no. 31, pp. 13052-13057, 2009.

[31] B. Kefas, J. Godlewski, L. Comeau et al., "microRNA-7 inhibits the epidermal growth factor receptor and the akt pathway and is down-regulated in glioblastoma," Cancer Research, vol. 68, no. 10, pp. 3566-3572, 2008.

[32] L. Jiang, X. Liu, Z. Chen et al., "MicroRNA-7 targets IGF1R (insulin-like growth factor 1 receptor) in tongue squamous cell carcinoma cells," Biochemical Journal, vol. 432, no. 1, pp. 199205, 2010.

[33] M. Peeters, T. Price, and J. L. Van Laethem, "Anti-epidermal growth factor receptor monotherapy in the treatment of metastatic colorectal cancer: where are we today?" Oncologist, vol. 14, no. 1, pp. 29-39, 2009.

[34] O. Saydam, O. Senol, T. Würdinger et al., "miRNA-7 attenuation in schwannoma tumors stimulates growth by upregulating three oncogenic signaling pathways," Cancer Research, vol. 71, no. 3, pp. 852-861, 2011.

[35] X. Zhao, W. Dou, L. He et al., "MicroRNA-7 functions as an antimetastatic microRNA in gastric cancer by targeting insulin-like growth factor-1 receptor," Oncogene, vol. 32, no. 11, pp. 13631372, 2013.

[36] S. Qu, X. Yang, X. Li et al., "Circular RNA: a new star of noncoding RNAs," Cancer Letters, vol. 365, no. 2, pp. 141-148, 2015.

[37] Y. Zhang, L. Yang, and L.-L. Chen, "Life without A tail: new formats of long noncoding RNAs," International Journal of Biochemistry \& Cell Biology, vol. 54, pp. 338-349, 2014.

[38] L.-L. Chen, "The biogenesis and emerging roles of circular RNAs," Nature Reviews Molecular Cell Biology, vol. 17, no. 4, pp. 205-211, 2016.

[39] F. Li, L. Zhang, W. Li et al., "Circular RNA ITCH has inhibitory effect on ESCC by suppressing the Wnt/ $\beta$-catenin pathway," Oncotarget, vol. 6, no. 8, pp. 6001-6013, 2015.

[40] C. E. Burd, W. R. Jeck, Y. Liu, H. K. Sanoff, Z. Wang, and N. E. Sharpless, "Expression of linear and novel circular forms of an INK4/ARF-associated non-coding RNA correlates with atherosclerosis risk," PLoS Genetics, vol. 6, no. 12, Article ID e1001233, 2010.

[41] D. Sayed, M. He, C. Hong et al., "MicroRNA-21 is a downstream effector of AKT that mediates its antiapoptotic effects via suppression of fas ligand," The Journal of Biological Chemistry, vol. 285, no. 26, pp. 20281-20290, 2010.
[42] L. P. Lim, N. C. Lau, P. Garrett-Engele et al., "Microarray analysis shows that some microRNAs downregulate large numbers of target mRNAs," Nature, vol. 433, no. 7027, pp. 769-773, 2005.

[43] S. Werfel, S. Nothjunge, T. Schwarzmayr, T.-M. Strom, T. Meitinger, and S. Engelhardt, "Characterization of circular RNAs in human, mouse and rat hearts," Journal of Molecular and Cellular Cardiology, vol. 98, pp. 103-107, 2016.

[44] F. Taibi, V. Metzinger-Le Meuth, Z. A. Massy, and L. Metzinger, "miR-223: an inflammatory oncomiR enters the cardiovascular field," Biochimica et Biophysica Acta (BBA)-Molecular Basis of Disease, vol. 1842, no. 7, pp. 1001-1009, 2014.

[45] Y.-S. Wang, J. Zhou, K. Hong, X.-S. Cheng, and Y.-G. Li, "MicroRNA-223 displays a protective role against cardiomyocyte hypertrophy by targeting cardiac troponin I-interacting kinase," Cellular Physiology and Biochemistry, vol. 35, no. 4, pp. 1546-1556, 2015.

[46] K. Wang, B. Long, F. Liu et al., "A circular RNA protects the heart from pathological hypertrophy and heart failure by targeting miR-223," European Heart Journal, vol. 37, no. 33, pp. 2602-2611, 2016.

[47] I. Murtaza, H.-X. Wang, X. Feng et al., "Down-regulation of catalase and oxidative modification of protein kinase CK2 lead to the failure of apoptosis repressor with caspase recruitment domain to inhibit cardiomyocyte hypertrophy," The Journal of Biological Chemistry, vol. 283, no. 10, pp. 5996-6004, 2008.

[48] B. V. Rodriguez, E. T. Malczewskyj, J. M. Cabiya, L. K. Lewis, and C. Maeder, "Identification of RNase-resistant RNAs in Saccharomyces cerevisiae extracts: separation from chromosomal DNA by selective precipitation," Analytical Biochemistry, vol. 492, pp. 69-75, 2016.

[49] S. Donath, P. Li, C. Willenbockel et al., "Apoptosis repressor with caspase recruitment domain is required for cardioprotection in response to biomechanical and ischemic stress," Circulation, vol. 113, no. 9, pp. 1203-1212, 2006.

[50] D. Mozaffarian, E. J. Benjamin, A. S. Go et al., "Heart disease and stroke statistics-2015 update: a report from the American Heart Association," Circulation, vol. 131, no. 4, pp. e29-e322, 2015.

[51] B. Swynghedauw, "Molecular mechanisms of myocardial remodeling," Physiological Reviews, vol. 79, no. 1, pp. 215-262, 1999.

[52] Y.-J. Zhao, J.-H. Wang, B. Fu et al., "Effects of 3-aminobenzamide on expressions of poly(ADP ribose)polymerase and apoptosis inducing factor in cardiomyocytes of rats with acute myocardial infarction," Chinese Medical Journal, vol. 122, no. 11, pp. 1322-1327, 2009.

[53] H.-H. Geng, R. Li, Y.-M. Su et al., "The circular RNA Cdrlas promotes myocardial infarction by mediating the regulation of miR-7 $\alpha$ on its target genes expression," PLoS ONE, vol. 11, no. 3, Article ID e0151753, 2016.

[54] B. Li, R. Li, C. Zhang et al., "MicroRNA-7a/b protects against cardiac myocyte injury in ischemia/reperfusion by targeting poly(ADP-ribose) polymerase," PLoS ONE, vol. 9, no. 3, Article ID e90096, 2014.

[55] D. E. Read, A. Gupta, Y. Ladilov, A. Samali, and S. Gupta, "miRNA signature of unfolded protein response in $\mathrm{H} 9 \mathrm{c} 2$ rat cardiomyoblasts," Cell \& Bioscience, vol. 4, no. 1, article 56, 2014.

[56] W. W. Du, W. Yang, Y. Chen et al., "Foxo3 circular RNA promotes cardiac senescence by modulating multiple factors associated with stress and senescence responses," European Heart Journal, 2016. 
[57] W. W. Du, W. Yang, E. Liu, Z. Yang, P. Dhaliwal, and B. B. Yang, "Foxo3 circular RNA retards cell cycle progression via forming ternary complexes with p21 and CDK2," Nucleic Acids Research, vol. 44, no. 6, pp. 2846-2858, 2016.

[58] A. Gschwendtner, S. Bevan, J. W. Cole et al., "Sequence variants on chromosome 9p21.3 confer risk for atherosclerotic stroke," Annals of Neurology, vol. 65, no. 5, pp. 531-539, 2009.

[59] S. Ye, J. Willeit, F. Kronenberg, Q. Xu, and S. Kiechl, "Association of genetic variation on chromosome 9p21 with susceptibility and progression of atherosclerosis: a populationbased, prospective study," Journal of the American College of Cardiology, vol. 52, no. 5, pp. 378-384, 2008.

[60] Y. Liu, H. K. Sanoff, H. Cho et al., "INK4/ARF transcript expression is associated with chromosome 9p21 variants linked to atherosclerosis," PLoS ONE, vol. 4, no. 4, Article ID e5027, 2009.

[61] J. Salzman, R. E. Chen, M. N. Olsen, P. L. Wang, and P. O. Brown, "Cell-type specific features of circular RNA expression," PLoS Genetics, vol. 9, no. 9, Article ID e1003777, 2013.

[62] J. L. Jacobs, K. Kieboom, S. Marino, R. A. DePinho, and M. Van Lohuizen, "The oncogene and polycombgroup gene bmi1 regulates cell proliferation and senescence through the ink $4 \mathrm{a}$ locus," Nature, vol. 397, no. 6715, pp. 164-168, 1999.

[63] R. Terranova, S. Yokobayashi, M. B. Stadler et al., "Polycomb group proteins Ezh2 and Rnf2 direct genomic contraction and imprinted repression in early mouse embryos," Developmental Cell, vol. 15, no. 5, pp. 668-679, 2008.

[64] J. L. Rinn, M. Kertesz, J. K. Wang et al., "Functional demarcation of active and silent chromatin domains in human HOX loci by noncoding RNAs," Cell, vol. 129, no. 7, pp. 1311-1323, 2007.

[65] J. Zhao, B. K. Sun, J. A. Erwin, J.-J. Song, and J. T. Lee, "Polycomb proteins targeted by a short repeat RNA to the mouse $\mathrm{X}$ chromosome," Science, vol. 322, no. 5902, pp. 750-756, 2008.

[66] Y.-C. Liu, J.-R. Li, C.-H. Sun et al., "CircNet: a database of circular RNAs derived from transcriptome sequencing data," Nucleic Acids Research, vol. 44, no. 1, pp. D209-D215, 2016.

[67] L. Qian, L. W. Van Laake, Y. Huang, S. Liu, M. F. Wendland, and D. Srivastava, "miR-24 inhibits apoptosis and represses Bim in mouse cardiomyocytes," The Journal of Experimental Medicine, vol. 208, no. 3, pp. 549-560, 2011.

[68] X. Wang, X. Zhang, X.-P. Ren et al., “MicroRNA-494 targeting both proapoptotic and antiapoptotic proteins protects against ischemia/reperfusion-induced cardiac injury," Circulation, vol. 122, no. 13, pp. 1308-1318, 2010.

[69] X. Wang, T. Liu, Z. Zhao, and G. Li, "Noncoding RNA in cardiac fibrosis," International Journal of Cardiology, vol. 187, no. 1, pp. 365-368, 2015.

[70] D. Baek, J. Villén, C. Shin, F. D. Camargo, S. P. Gygi, and D. P. Bartel, "The impact of microRNAs on protein output," Nature, vol. 455, no. 7209, pp. 64-71, 2008.

[71] D. W. Thomson, C. P. Bracken, and G. J. Goodall, "Experimental strategies for microRNA target identification," Nucleic Acids Research, vol. 39, no. 16, pp. 6845-6853, 2011.

[72] G. Kalozoumi, M. Yacoub, and D. Sanoudou, "MicroRNAs in heart failure: small molecules with major impact," Global Cardiology Science \& Practice, vol. 2014, no. 2, article no. 30, 2014.

[73] F. He, P. Lv, X. Zhao et al., "Predictive value of circulating miR328 and miR-134 for acute myocardial infarction," Molecular and Cellular Biochemistry, vol. 394, no. 1-2, pp. 137-144, 2014.
[74] Y. Yao, J. Du, X. Cao et al., "Plasma levels of microRNA499 provide an early indication of perioperative myocardial infarction in coronary artery bypass graft patients," PLOS ONE, vol. 9, no. 8, Article ID e104618, 2014.

[75] H. Gao, R. R. Guddeti, Y. Matsuzawa et al., "Plasma levels of microRNA-145 are associated with severity of coronary artery disease," PLoS ONE, vol. 10, no. 5, Article ID e0123477, 2015. 


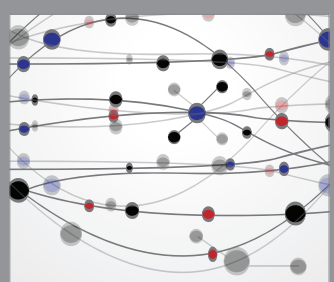

The Scientific World Journal
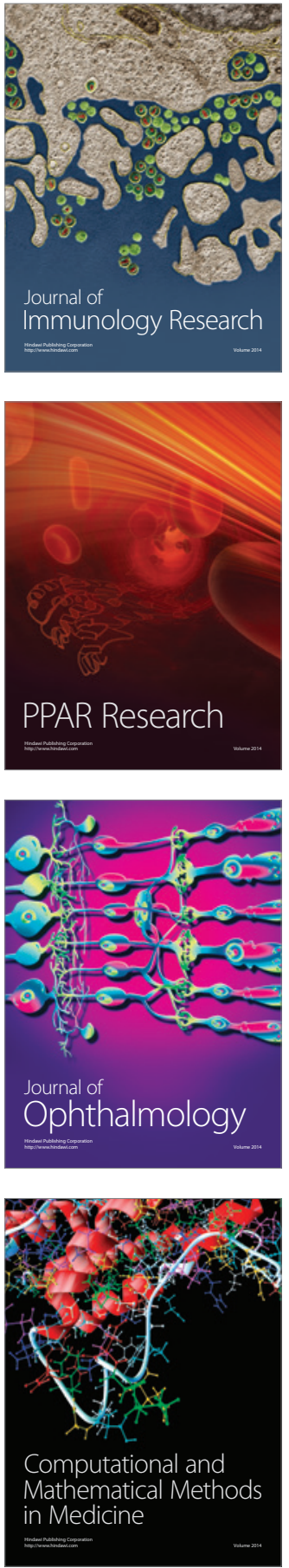

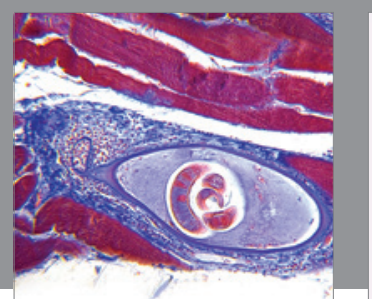

Gastroenterology Research and Practice
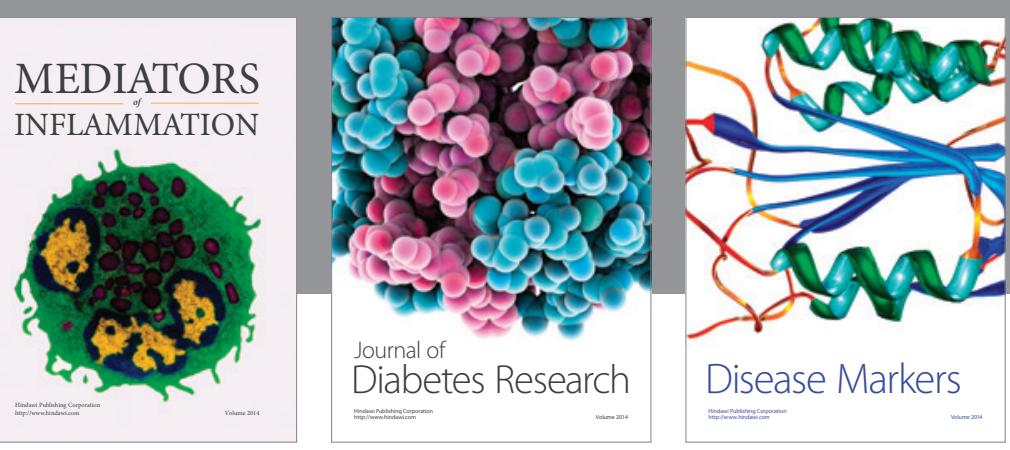

Disease Markers

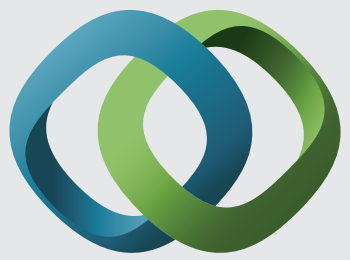

\section{Hindawi}

Submit your manuscripts at

https://www.hindawi.com
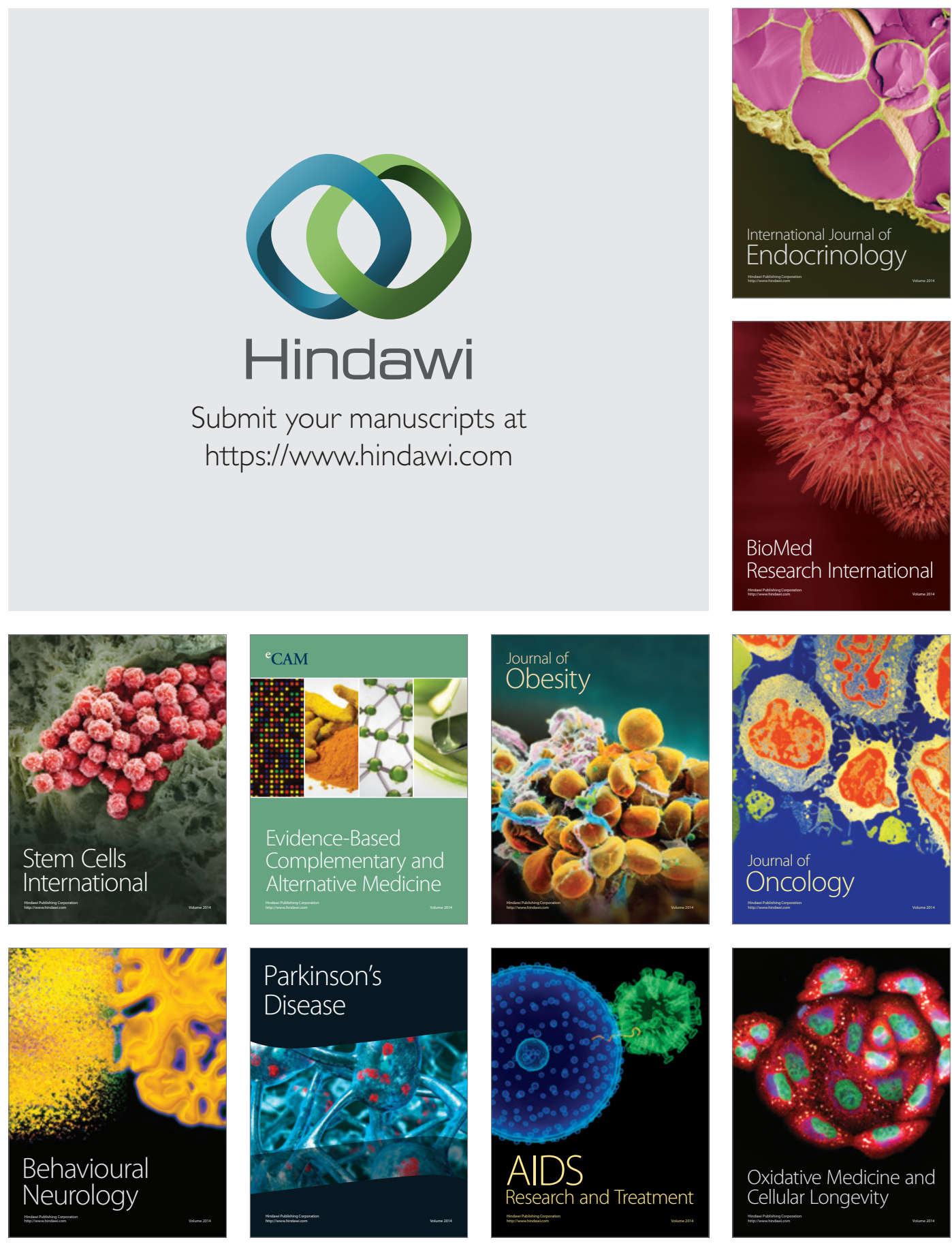